\title{
DE L'INSECURITE SUR LA VILLE DE LUBUMBASHI SUITE AU TAUX ELEVE DE CHOMAGE
}

\author{
Par KISIMBA BONDO Kis, KABULO CHUNDA Raoul, KABOZYA WA KA- \\ BOZYA Joseph et MUBAKA KATALAY Véro*
}

\section{INTRODUCTION}

La République Démocratique du Congo fait face à une insécurité alarmante et les provinces et villes ne sont pas épargnées chaque jour les populations sont victimes de vol, viol, meurtre, coups et blessures et cela place la population dans une situation de peur car, les gens passent des nuits blanches pour garantir une sécurité à leurs vies physiques et à leurs à leurs biens.

Cependant, la ville de Lubumbashi, une ville qui reçoit tout le monde n'est pas épargnée, car elle est aussi victime chaque nuit. Il se dégage que l'absence de l'emploi est à la base de cette insécurité, nul n'ignore que plus de $71,3 \%$ de la population Lushoise est au chômage et vit de la manière incertaine et de la mendicité, il suffit seulement d'acheter un bien mobilier, faire une transaction en plein jour et devant les inconnus, ramener chaque soir un sachet des pains à la maison; être bien habiller chaque jour vous devenez un cible incontournable des bandits.

La constitution du 18 février 2006, telle que modifiée par la loi №11/002 du 20 Janvier 2011, portant révision de certains articles dispose que : « La personne humaine est sacrée : l'Etat à l'obligation de la respecter et de la protéger,toute personne a droit à la vie, à l'intégrité physique ainsi qu'au libre développement de sa personnalité dans le respect de la loi de l'ordre public, du droit d'autrui et des bonnes mœurs. ${ }^{1}$

Et l'article 15 de la même constitution dispose que : « Les pouvoirs publics veillent à l'élimination des violences sexuelles. Sans préjudices des traités et accords internationaux, toute violence sexuelle faite sur toute personne, dans l'intention de déstabiliser, de disloquer une famille est de faire disparaitre tout un peuple est érigée en crime contre l'humanité puni par la loi ». ${ }^{2}$

De la compréhension de ce deux dispositions, il y a lieu d'affirmer que le droit humanitaire est violé chaque jour dans la ville de Lubumbashi suite à l'insécurité, car les femmes mariées sont violées en présence de leurs maris et les familles sont divisées, les jeunes filles violées en présence de leurs parents avec l'objectif de disloquer les familles, car c'est sans conscience et pudeur de faire le rapport sexuel avec la femme de quelqu'un en sa présence

* Tous Assistants à la Faculté de Droit de l'Université de Lubumbashi.

1 Article 16 de la constitution du 18 Février 2006, telle que modifiée par la loi n $11 / 002$ du 20 Janvier 2011, portant révision de certains articles.

2 Article 15 de la même constitution (RDC). 
ou pour une fille en présence de son père.Plus criminel encore obligé à un père de coucher avec sa propre fille, ou un garçon de coucher avec sa propre mère.

Que dire d'autres, les Hommes sont tués chaque jour (Hommes et femmes, petits et vieux) sans pitié, ni amour du prochain, or la vie humaine est sacrée, personne n'a le droit de mettre fin à la vie de l'autre. Un constat malheureux est celui devoir que l'Etat congolais est passif plonger dans la batailles des postes et à la recherche des intérêts privés, car ceux qui sèment l'insécurité collaborent semble-t-il avec les autorités judiciaires et militaires. Les peuples exigent une chose, la sécurité de leurs vies physiques et de leurs biens.

\section{A. ORIGINE DE L'INSECURITE A LUBUMBASHI}

Notre constat part des années 2000 à 2007 au moment où à Lubumbashi, les gens pratiquaient beaucoup plus l'exploitation minière artisanale.

En effet, pendant cette période, travailleur comme non - travailleur, tous vivaient mieux et personne ne pouvait envier l'autre. Il a fallu voir certains jeunes à l'âge de scolarité enterré leurs études pour aller dans des carrières même les mineurs. Pendant cette période tout le monde avait l'argent et l'insécurité ne se faisait pas remarquer, du moins il y avait un calme et tout le monde vivait dans la quiétude, tous les gens étaient à l'aise. Il fallait voir l'exode rural était accru à cause de l'exploitation minière artisanale. Tout celui qui était chômeur pensait que la carrière était la seule activité qui payait mieux.

Déjà à ce niveau, les autorités politico-administratives de l'époque ont laissé une porte ouverte à l'insécurité dans la ville de Lubumbashi étant donné qu'il n'y avait pas de mesures d'encadrement, n'importe qui pouvait aller à la carrière, jeune garçon, jeune fille, femmes mariées, bref tout le monde. Les mêmes autorités de l'époque n'ont pas pensée à l'après carrière minière.

Lorsque maintenant l'Etat devrait concéder les différentes carrières qui sont autour de la ville de Lubumbashi aux exploitants expatriés et professionnels, tout en promettant aux creuseurs artisanaux l'engagement dans différentes firmes minières, la plupart d'entre-deux par manque d'instruction scolaire n'ont pas pu être embauchés. Cela a fait des conséquences négatives sur la ville de Lubumbashi.

A titre illustratif, le fait pour les femmes mariées d'aller à la carrière, cela a entrainé beaucoup des divorces, pour les jeunes filles et garçons. Les mariages précoces. Tout ceci c'était l'ouverture de l'insécurité à Lubumbashi. Signalons également que parmi les jeunes gens qui avaient l'âge entre 20 ans et 30 ans qui étaient dans les carrières environnantes la ville de Lubumbashi, nombreux étaient des ex-militaires du régime Laurent Désiré KABILA.

Les femmes qui avaient divorcés elles ont fait des enfants en désordre et ces enfants la plupart d'entre eux ont été abandonné à cause du cout dur de la vie, comme conséquence ces enfants ont été exposés à des risques graves, d'où une autre brèche qui s'est ouverte pour permettre à l'insécurité d'élire domicile dans la ville de Lubumbashi. Disons que c'est à partir de ce moment que l'Etat devrait jouer son rôle d'encadreur tout en recadrant ces 
activités d'exploitation minière artisanale, sensibilisé ces creuseurs artisanaux sur les bienfaits (bien - fondé) de l'agriculture.

Pendant cette période, l'Etat a été absent en ce qui concerne l'encadrement de la population Lushoise.

\section{B. SITUATION SECURITAIRE DANS LES PERIPHERIES DE LA VILLE DE LUBUMBASHI}

Si à Lubumbashi la population ne sait pas passer une nuit sans être inquiétée, ce n'est pas le cas pour ceux qui sont à SHINDAIKA, village à plus de $20 \mathrm{Km}$ à l'Est de la ville de Lubumbashi.

En effet, dans ce milieu de la périphérie de la ville de Lubumbashi, certaines personnes ont même des maisons de fortune (case, hutte), de fois lesdites maisons n'ont même pas de porte. Nous avons mené une étude et comme résultat nous avons trouvés comme conclusion que chaque personne du village a au moins un champ pour l'auto-alimentation; un champ pour vendre les fruits de la récolte afin de subvenir à ses besoins. Presque chaque ménage a au moins un moyen de locomotion et certains ont même des moulins. Si nous citons tous ces exemples, c'est parce que dans cette partie du territoire national, il y a la sécurité des personnes et de leurs biens, malgré la non présence des éléments de l'ordre.

Il est rare de trouver un élément de l'ordre dans cette partie et même un poste de police.

Ceci nous pousse à dire que le fait pour tout le monde d'avoir une source de revenu, avoir une occupation vivrière, fait à ce que la sécurité s'impose elle -même dans cette contrée. Disons également que, malgré la non scolarisation des enfants que nous trouvons là-bas, leurs parents leurs apprennent les activités champêtres, et volé, tué, violé est un tabou.

Il est vrai qu'on a noté quelques cas négatifs (néfastes) c'est le cas notamment des mariages précoces, mais cela n'a pas un impact négatif sur la sécurité et même la non - scolarisation n'a pas non plus un impact négatif sur ladite sécurité.

\section{LES CAUSES DE L'INSECURITE A LUBUMBASHI}

\section{LA PAUVRETE}

La pauvreté est aujourd'hui à la base de manière incontournable de l'insécurité dans la ville de Lubumbashi, caractérisé par le chômage et le non-paiement de la minorité qui travaillent.

Un député national tire la sonnette d'alarme sur la montée de l'insécurité et la pauvreté qui ont élu domicile à Lubumbashi, chef-lieu de la province du Haut-Katanga.

Dans un entretien avec le potentiel l'élue de Lubumbashi s'exprime en ces termes « Je trouve paradoxale le fait que la province du Haut-Katanga regorge de toute une diversité de 
ressources naturelles et agricoles alors que les populations locales et de cette région cuprifère croupissent dans une pauvreté extrême. ${ }^{3}$

Nous déduisons que, le chômage est à la base de la pauvreté qui en suite pousse les gens à réfléchir autrement de la manière à faire du mal aux autres une situation que les autorités n'ont pas jusqu'ici maitrisé pour apporter la paix et la sécurité, ainsi que la confiance dans la population.

Aujourd'hui, l'insécurité s'empire sous l'œil passif des autorités du pays. Faute d'emploi aux citoyens et une prise en charge responsable des délinquants. Les femmes, les jeunes filles seront violées par des criminels devant leurs maris et pères, en plus les hommes seront tués, les enfants et bébés égorgés.

Il n'existe plus un quartier à Lubumbashi ou il fait beau vivre, nous observons un manque total d'humanisme s'est donc installé dans la ville caractérisée par la peur toutes les nuits ou personnes n'est sur du lendemain et face à cette situation la population à ses risques passent nuit à la belle étoile car, elle n'a ni arme, ni objet conséquent à ripostée à la menace des bandits, nous vivons dans un Etat sans foi, amour et sans justice.

Selon la Banque Mondiale dans son rapport de 2012 sur la situation économique de la République Démocratique du Congo, pense que 71, 3\% de congolais majoritaire sont des pauvres, alors qu'une minorité vit dans l'opulence et avec cette situation il n'y a pas moyen de lutter contre l'insécurité économique et physique de la population). ${ }^{4}$

Selon ce classement, les congolais vit avec moins d'un dollars américain par jour. Pour remédier à cette situation, son organisation accompagne les mamans maraichères en leur octroyant des semences et les engrais et puis, avec des psychologues, elle a écouté et encadré une centaine de jeunes désœuvrés qui sont devenus des micros entrepreneurs.

Il faut noter que tant qu'il n'y a aura pas du travail et de la prise en charge responsable, nous allons continuer soigner les femmes violées, à enterrer les hommes et les bébés égorgés, nous devons donc nous attaqués aux causes.

L'article 36 de la constitution du 18 février 2006 dispose que : « Le travail est un droit et un devoir sacré pour chaque congolais. L'Etat garantit le droit au travail, la protection contre le chômage et une rémunération équitable et satisfaisante assurant au travailleur ainsi qu'à sa famille une existence conforme à la dignité humaine, complétée par tous les autres moyens de protection sociale notamment la pension de retraite et la rente viagère ${ }^{5}$

3 https://lepotentielonline.net/2019/3/27/Haut-Katanga-l'insécurité-et-la pauvreté-sévissent à Lubumbashi, page consultée le 10/06/2019 à 07h27.

4 https://lepotentielonline.net/2019/3/27/Haut-Katanga-l'insécurité-et-la pauvreté-sévissent à Lubumbashi, page consultée le 10/06/2019 à 07h53.

5 Article 36 de la constitution du 18 Février 2006, telle que modifiée par la loi n¹1/002 du 20 Janvier 2011, portant révision de certains articles. 


\section{L'ETAT EST PASSIF FACE A LA SITUATION ACTUELLE DE L'INSECURITE A LUBUMBASHI}

La passivité de l'Etat est constaté lorsque les bandits sont arrêtés ils sont vites relâché et donc un manque des sanctions et d'un Etat de droit. D'aucuns estiment que ceux qui sont à la base de l'insécurité à Lubumbashi sont les policiers et soldats qui opèrent avec les civils en leur fournissant les matériels (armes et tenues, ...) parce que les soldats et policiers de la République reçoivent un solde qui est insuffisant et donc impossible de faire face à la vie étant père d'une femelle. Car souvent les biens volés sont retrouvés chez les militaires et policiers; les bandits arrêtés sont soient en tenue militaire, soit en tenue policière une indiscipline qui ne dit pas son nom, l'Etat est démissionnaire surtout le plan il y a donc lieu de proposer la réforme de l'Etat.

Les mécanismes de contrôle doivent être consolidés par la création de services de renseignement et de sécurité tels que la détection militaire des actions anti-partie avec la nouvelle technologie de la communication. ${ }^{6}$

La sécurité procure la paix et la stabilité ainsi les deux conduisent au développement d'un pays. ${ }^{7}$

La sécurité est la clef d'ouverture dans toutes les sociétés humaine, les personnes ont besoin de la sécurité physique et de leurs biens gage de la stabilité économique. L'Etat doit revenir à la raison pour prendre à main sa responsabilité régalienne. Dans un Etat moderne, l'Etat doit être aux côtés de sa population est non pas la population aux cotés de l'Etat une confusion finie toujours par une instabilité et une auto-prise en charge qui est souvent moindre face aux menaces de criminels.

Cependant, la désolation est que l'Etat congolais est devenu plus faibles et ces animateurs très forts, l'on se croirait dans un état sans loi, ni justice.

\section{LA NON SCOLARISATION DES JEUNES ET LA NON IDENTIFICATION DE LA POPULATION PAR LES SERVICES DE L'ETAT}

a) La non scolarisation des jeunes

Un homme sans instruction est un manque à gagner pour son pays.

Déjà l'article 42 de la constitution du 18 Février 2006 dispose que : « Les pouvoirs publics ont l'obligation de protéger la jeunesse contre toute atteinte à sa santé, à son éducation et à son développement intégrale. ${ }^{8}$

6 FILIP REYNT JENS, La grande guerre Africaine, instabilité, violence et déclin de l'Etat en Afrique Centrale, Ed. Les belles lettres, Paris, 2012.

7 NGOTO NGOIE NGALINGI, L'essentiel du Droit Pénal Congolais, PUC, Kinshasa, 2018, p. 197.

8 Article 42 de la constitution du 18 Février 2006, telle que modifiée par la loi n $11 / 002$ du 20 Janvier 2011, portant révision de certains articles. 
Et l'article 43 de la même constitution dispose que : « Toute personne a droit à l'éducation scolaire. ${ }^{9}$

Il est dégagé ce qui suit, que l'éducation et l'instruction sont à la base du développement d'un Etat, l'Etat doit compter sur une bonne jeunesse qui constitue l'espoir d'un Congo émergent.

Mais dans la suite de nos constats, plusieurs enfants ne sont pas scolarisés à cause de la pauvreté de leurs parents, mais surtout de l'irresponsabilité de l'Etat congolais sur l'encadrement de la jeunesse.

Nous retrouvons les jeunes enfants en âge de scolarité aux angles des avenues à travers la ville de Lubumbashi qui sèment de l'insécurité de manière permanente sous l'œil passif des autorités politiques et administratives du pays en général et en particulier ceux de la province et de la ville de Lubumbashi qui parfois donne même de l'argent à cette jeunesse.

Peut-on dire que l'insécurité à Lubumbashi est entretenue par les autorités? Répondre qui peut.

Aujourd'hui il est difficile de rouler sa voiture à 18 Heures, voir même à 17 heures les vitres baisser, non seulement avec sa voiture même en transport en commun, plusieurs de nos compatriotes ont étaient victime de vol des téléphones qui les avaient coutés mais volés par un enfant en désespoir pour aller revendre à un prix dérisoire. Il ne fait plus beau vivre à Lubumbashi, les jeunes filles sont violées pendant la journée, leurs sacs emporter et souvent elles en sortent avec des blessures suite à la résistance éphémère, il faut donc marcher en groupe lorsqu'il faut passer sur une avenue où résident les jeunes que nous appelons vagabonds.

b) La non identification de la population par les services de l'Etat

La gestion d'une cité demande la maitrise des administrés, connaitre leurs activités, leurs compositions familiales, pour prétendre leurs offrirent une sécurité nécessaire.

Ainsi le gouvernement provincial de Tanganyika a lancé un communiqué sur le recensement obligatoire de la population. "Il est porté à la connaissance de toute la population de Kalemie que sous le Haut patronage de son Excellence Monsieur le Gouverneur de la Province, Le Ministre Provincial de l'intérieur affaires coutumiers organises en collaboration avec la mairie de Kalemie un recensement individuel au cours duquel chaque habitant aura à remplir une fiche de renseignement individuel et une fiche de composition de la cellule (NYUMBA 10).

Ce recensement étant obligatoire et gratuit aucune taxe ne sera exigée lors de cette opération et tout déménagement doit au préalable requérir l'approbation du chef de quartier. ${ }^{10}$

9 Article 43 de la constitution du 18 Février 2006, telle que modifiée par la loi n¹1/002 du 20 Janvier 2011, portant révision de certains articles.

10 Communique officiel du gouvernement provincial de Tanganyika, ${ }^{\circ} 009 / 000 / \mathrm{CAB} / \mathrm{MININTER}-$ SECDA/TANG/2019. 
Une initiative louable parce que la ville aura l'effectif normal et saura qui vit ou et qui vient d'arriver pour quel motif et chez qui seul de cette façon que nous pouvons lutter contre l'insécurité.

Il faut noter que l'identification de la population n'est pas en contradiction avec l'article 30 de la constitution du 18 Février 2006 qui dispose que : " Toute personne qui se trouve sur le territoire national a le droit résidence, d'y circuler librement, d'y fixer sa résidence; de le quitter et d'y revenir dans les conditions fixées par la loi. Aucun congolais ne peut être ni expulsée du territoire de la République, ni être contraint à l'exil, ni être forcé à habiter hors de sa résidence habituelle $»{ }^{11}$

Les mécanismes d'identifications sont un moyen de renforcement de sécurisation de la population et non pas un moyen discriminatoire. Mais le fait que les populations font de l'exode des provinces et villes sans un contrôle préalable nous sommes victimes de l'insécurité, car les autorités ne savent pas la situation ni judiciaire, ni professionnelle, ni familiale de ceux qui viennent et qui font des déplacements d'une province (ville) à une autre.

L'exode est justifié par le fait que les gens font de déplacement à la recherche d'une vie meilleure et la ville de Lubumbashi paie le frais parce que ne disposant pas d'un mécanisme de contrôle et d'identification des populations qui habitent son sol.

\section{SUGGESTIONS ET/OU PROPOSITIONS}

La vie humaine est sacrée et l'Etat doit une protection sérieuse à sa population qui paie les impôts malgré les maigres moyens qu'elle dispose.

Mais tant qu'il n'y aura pas l'implication de l'Etat en matière sécuritaire et surtout la sécurité économique et l'encadrement de la jeunesse de manière responsable nous allons constituer à soigner les femmes violées et nous allons enterrer les hommes chaque jour dans notre ville, c'est ici ou nous appelons la bonne conscience de notre Etat.

Face à cette situation des désolations et pour y apporter une solution durable dans l'espoir de mettre fin à une insécurité sans humanisme et pleine de haine, nous proposons les solutions suivantes :

- Que l'Etat crée de l'emploi;

- Que l'Etat retire les policiers et soldats dans les quartiers civils;

- Que l'Etat paie correctement les hommes en uniforme;

- Que l'Etat retire les jeunes enfants de la rue;

- Que l'Etat autorise de manière officielle l'identification des populations dans chaque provinces lors du déplacement;

- Que l'Etat scolarise les jeunes enfants en âge de scolarité pour préparer un bel avenir pour eux;

11 Article 30 de la constitution du 18 Février 2006, telle que modifiée par la loi n¹1/002 du 20 Janvier 2011, portant révision de certains articles. 
- Que la sanction soit très lourde pour les criminels de viols et de tueries des hommes et des bébés;

- Que l'Etat encadre les familles à l'agriculture;

- Que l'Etat paie conséquemment les travailleurs et impose aux entreprises privées de faire la même chose.

- Faire un planning familial.

Viola de manière synthétique nos propositions qui peuvent être une solution à la lutte contre l'insécurité que nous décrions aujourd'hui dans la ville de Lubumbashi.

\section{CONCLUSION}

Nous avons voulu dans cette étude, nous faire une idée sur la manière d'éradiquer l'insécurité à Lubumbashi, insécurité due au taux élevé du chômage. Il a été révélé que si les autorités politico-administratives subventionnent l'agriculture et sensibilisent tous les chômeurs de la ville de Lubumbashi sur le bienfondé de celle -ci (agriculture), on diminuera le chômage et par conséquent, on éradiquerait cette dernière. Nous avons soutenu notre position en faisant une petite étude comparative avec une zone périphérique de Lubumbashi (Village SHINDAIKA), où il a été observé la sécurité sans qu'il n'y ait même pas les éléments de l'ordre.

En effet, il a été démontré que, suite aux travaux champêtres, ces gens vivent mieux et par conséquent, ils sont dans les quiétudes et donc ils sont dans la sécurité, malgré quelques cas néfastes soulevés, c'est le cas notamment des mariages précoces et l'alcoolisme.

Enfin, nous avons formulé quelques recommandations pouvant aider à l'éradication de l'insécurité à Lubumbashi.

\section{BIBLIGRAPHIE}

\section{TEXTES DES LOIS}

1. Constitution du 18 Février 2006; telle que modifiée par la loi n¹1/002 du 20 Janvier, 2011, portant révision de certaines articles

\section{OUVRAGES}

1. NGOTO NGOIE NGALINGI, l'essentiel du Droit Pénal Congolais, PUC, Kinshasa, 2018. éd. Les belles lettres, Paris 20102

2. BACHMANN, $P$ : Communiquer avec la presse écrite et audiovisuelle, $3^{\text {ème }}$ édition, victoires, Paris 2004

3. WEST PHALEN, M.H; Communication, le guide de la communication d'entreprise, $3^{\text {ème }}$ édition, Dunod, Paris 1998 
4. FILIP REYNT JENS, La grande guerre Africaine, instabilité, violence et déclin de l'Etat en Afrique Centrale, Ed. Les belles lettres, Paris, 2012.

\section{WEBOGRAPHIES}

1. https://leptentielonline.net 2019/3/27/Haut-Katanga - Lubumbashi-insécurité - et- la pauvreté, page consultée le 10/06/2019 à 07h27'

2. http://lepotentielleune.net /2019/3/2017/Haut-Katanga - Lubumbashi-L'insécurité et pauvreté : page consultée le 10/6/2019 à 07h53.

\section{AUTRES SOURCES}

1. Communique officiel du gouvernement provincial de Tanganyika, $n^{\circ} 009 / 000 / C A B / M I N I N T E R S E C$ DA/TANG/2019 